\title{
Excitation Mechanism in Laser-Induced Plasma at Atmospheric Air Pressure
}

\author{
Jojor Lamsihar Manalu ${ }^{1}$, Koo Hendrik Kurniawan ${ }^{2, *} \&$ Tjung Jie Lie ${ }^{2}$ \\ ${ }^{1}$ Graduate Program in Opto-Electrotechniques and Laser Applications, Faculty of \\ Engineering, University of Indonesia, 4 Salemba Raya, Jakarta 10430, Indonesia. \\ ${ }^{2}$ Research Center of Maju Makmur Mandiri Foundation, 40 Srengseng Raya, \\ Kembangan, Jakarta Barat 11630, Indonesia
}

\begin{abstract}
A special interferometric technique has been devised on the basis of rainbow refractometry without the use of an additional and delicate amplitudesplitting setup. This new technique was used for the characterization of plasma induced by a Q-switched Nd-YAG laser on zinc samples under atmospheric air pressure. An unmistakable signal of the density jump was detected simultaneously with the observation of the emission front signal. It was proved that the emission front and the front of the shock wave coincided and moved together with time at the initial stage of the secondary plasma expansion. However, at a later stage, the emission front began to separate from and left behind the shock wave front propagating in the surrounding air. With the use of zinc sample, the experimental results showed that the separation of the emission front and shock wave front took place at about $4 \mathrm{~mm}$ above sample surface for laser energy of $26 \mathrm{~mJ}$. It was also found that the separation time increased by increasing the power density which further supported shock wave model. Analysis of the data of the shock front movement along with the emission characteristics has led us to the conclusion that models other than the shock wave model, such as the gas breakdown model, should be excluded, at least for a zinc sample, as not satisfactorily explaining the excitation process in the secondary plasma generated at atmospheric air pressure of 760 Torr.
\end{abstract}

Keywords: laser-induced plasma, shock wave plasma, excitation stage, cooling stage,

\footnotetext{
"author to whom correspondence should be sent:

Koo Hendrik Kurniawan, Research Center of Maju Makmur Mandiri Foundation, 40 Srengseng Raya, Kembangan, Jakarta Barat 11630, Indonesia, Tel: 62-21-5867663, 62-21-5867660, Fax: 62-21-5867670 or 62-21-5809144 E-mail: kurnia18@.cbn.net.id,

Homepage: http://www.mmm.or.id
} 


\section{$1 \quad$ Introduction}

The study of Laser Atomic Emission Spectrochemical Analysis (LAESA) was first conducted by Brech et al [1] in 1962. The resulted LAESA technique has become a most typical spectroscopic application of laser, and many studies have been conducted on this subject, but mainly from the point of view of practical applications. The basic understanding on the physical process of excitation of the atoms gushing out from the target has nevertheless remained less than completely settled. This excitation process is very important not only for the emission spectrochemical analysis, but also for other applications employing laser ablation, such as laser processing, laser induced film deposition, and laser induced chemical reaction and so on. Once the emission process following the ablation process is fully understood, the atomic emission can be used for monitoring the ablation process in each case of application, and is hence useful for effective control of the related process.

Nowadays the development of LAESA is known to follow two major directions. One is the high pressure type, usually called Laser Induced Breakdown Spectroscopy (LIBS) [2-9]. In this method, a laser of high peak power with short duration such as Nd-YAG laser is focused onto the sample at atmospheric pressure. In order to remove the disturbing background spectrum coming from the high temperature and high density plasma, gated optical multichannel analyzer (OMA) is used in the detection system. Another version of LAESA is the low pressure type [10-14]. In this case, the laser plasma is produced under reduced pressures of surrounding gas aiming at the reduction of the background emission intensity in the spectrum.

In a series of studies on the plasma generation process under reduced pressure [15-25], we found that a plasma having characteristics favorable to spectrochemical analysis can actually be generated by using a pulsed laser with short duration, such as the nitrogen laser, carbon dioxide laser, excimer laser and Nd-YAG laser, when the pressure of the surrounding gas is reduced to around 1 Torr. In these cases, the plasma invariably consists of two distinct parts. The first part, which is called the primary plasma, occupies a small area and gives off intense continuous emission spectra for a short time just above the surface of the target. The other part, called the secondary plasma, expands with time around the primary plasma with near-hemispherical shape, emitting sharp atomic spectral lines with negligibly low background. The secondary plasma is free from undesirable self-absorption effect and satisfies a good linear relationship between the content in the sample and its emission intensity.

One of the main difficulties in the study of the ablation process is related with its highly transient nature as well as its exceedingly small space. Consequently, 
the ordinary spectroscopic techniques are rendered inadequate for its investigation. However, by means of a unique technique developed by us, which made possible the detection of time-resolved spatial distribution of the emission intensity, we have demonstrated that this secondary plasma was excited by the shock wave generated in the surrounding gas, while the primary plasma acted as an initial explosion energy source.

In a previous paper [26-27], we have offered another strong evidence in support of our shock wave model using a special interferometric technique to detect density jump due to the propagation of the shock wave. The density jump was detected simultaneously with the observation of emission front of the secondary plasma, demonstrating the coincidence of the emission front and the front of the shock wave, which move together with time at the initial stage of the secondary plasma expansion.

In spite of the detailed understanding described above, about laser plasma generated at low surrounding gas pressures, the fundamental mechanism of laser plasma generated at the atmospheric pressure has yet to be unraveled despite its remarkable popularity as a tool for spectrochemical analysis. In order to look for clues on this mechanism, we have carried out a detailed experiment employing a surrounding gas at 760 Torr. Time resolved measurement along with the detection of density jump were performed on the laser plasma produced at this air pressures in the hope of revealing new dynamical features which were hitherto unheeded. It will be shown that the analysis of these features led to a strong indication of the occurrence of secondary plasma even in the high pressure surrounding air.

\section{Experimental Procedure}

Figure 1 shows the experimental setup used in this work. A Nd-YAG laser (Quanta Ray, GCR-12S, $400 \mathrm{~mJ}, 8 \mathrm{~ns}$ ) was employed. The energy of the laser irradiation was varied with a set of filters, and the laser was operated under the Q-switched mode. The laser was run at a repetition frequency of $10 \mathrm{~Hz}$, and the shot-to-shot fluctuation of the laser was approximately $3 \%$. The laser beam was focused by a multilayer lens $(\mathrm{f}=100 \mathrm{~mm})$ through a quartz window onto the surface of the target.

The samples were placed in a vacuum tight metal chamber $(11 \mathrm{~cm} \mathrm{x} 11 \mathrm{~cm} \mathrm{x}$ $12.5 \mathrm{~cm}$ ), which could be evacuated with a vacuum pump and filled with air at the desired pressure. The air flowing through the chamber was regulated by a needle valve in the air line and a valve in the pumping line. The chamber pressure was measured precisely with the use of an absolute digital vacuum- 
meter. A zinc plate (Rare Metallic Co., 99.99\%, 0.4 mm thickness) was used as target in all the experiments.

The radiation of the laser-induced plasma was observed through the top side of the chamber via a mirror, and the image was plotted $1: 1$ by the aid of a quartz lens $(\mathrm{L} 2, \mathrm{f}=150 \mathrm{~mm}$ ) onto the entrance plane of the monochromator (SPEX M750 , Czerny-Turner configuration, focal length $750 \mathrm{~mm}$ with 1,200 grooves $/ \mathrm{mm}$ blazed at $500 \mathrm{~nm}$ ). The electrical signal from the photomultiplier (Hamamatsu IP-28) was fed through a $500 \Omega$ resistor [its resistance capacitance (RC) time constant was $30 \mathrm{~ns}$ ] into the first channel of digital sampling-storage scope (HP, model 54601-A, $500 \mathrm{MHz}$ ), and data collection was performed with a printer after passing through an RS-232 interface module. The trigger signal of the digital sampling-storage scope was provided from a fraction of the laser light by means of a PIN photodiode.

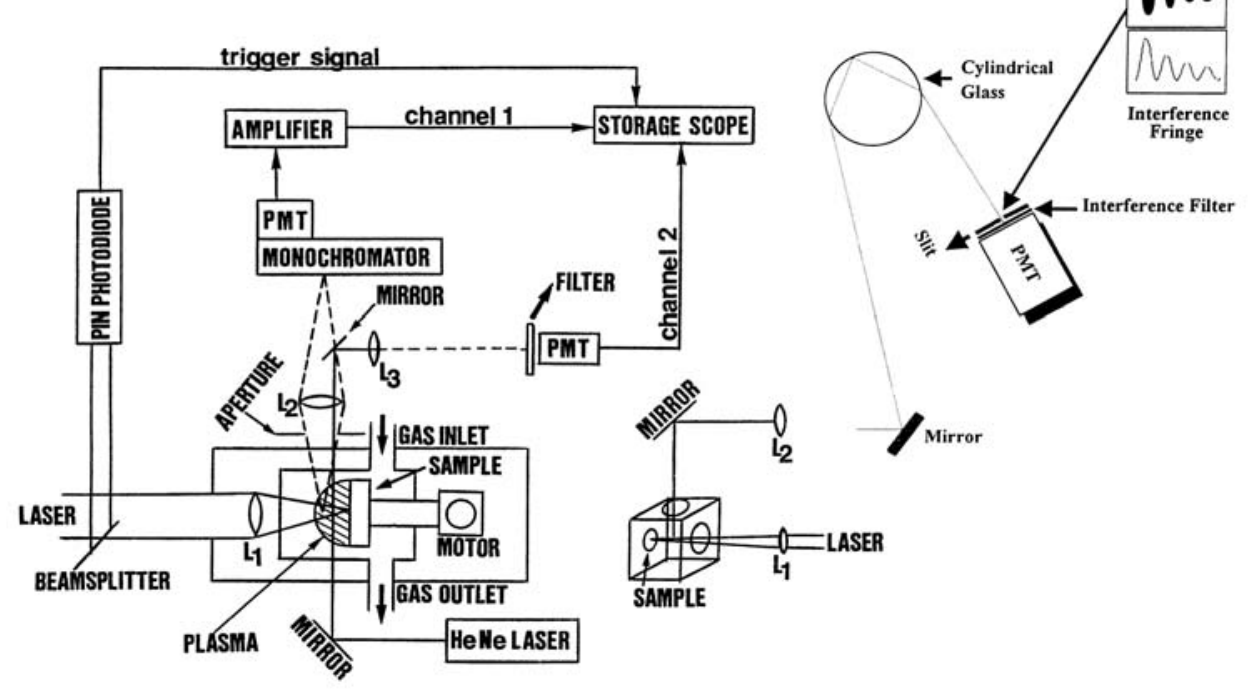

Figure 1 Experimental Setup.

For density jump measurements, a He-Ne laser was used, and the He-Ne laser light was sent perpendicular to the YAG light into the expansion region of the laser plasma by using a mirror. The outgoing probe beam was then sent into the cylindrical glass with a $60 \mathrm{~mm}$ diameter. A photomultiplier tube (PMT) (Hamamatsu R-1104) was positioned to detect the light coming out from the glass. Electrical signal coming from the PMT was analyzed by means of the second channel of the digital sampling-storage scope after the light passed 
through a resistor of $500 \Omega$ (its RC time constant was 30 ns). A vertical slit was used in front of the PMT for detecting the probing He-Ne laser light in order to avoid disturbances from other unwanted light sources, which were further blocked by an interference filter for the He-Ne laser wavelength placed behind the slit.

As shown in the inset of Fig. 1, the probe beam that emerges from the cylindrical glass appears as interference fringes. This interference effect is readily explained by the fact that the actual laser light is not a single ray, but a beam consisting of many rays with a cross section of around 1 to $2 \mathrm{~mm}$. Except for the central ray, all the other rays are slightly shifted from the condition of minimum deviation. These rays spread out and expand to some extent as they emerge from the cylindrical glass. Interference takes place among these rays and produces bright fringes in the directions which satisfy the phase-matching condition. Before one performs the measurement, the mirror in front of the cylindrical glass should be properly adjusted so that the He-Ne laser light will undergo a minimum deviation in passing through the cylindrical glass. Under this condition, the highest visibility of the interference fringes will be secured, as illustrated in the inset of Fig. 1. One of these fringes (second minimum) will then employed as a marker for the following measurement. The separation between adjacent fringe in the absence of the laser plasma is about $100 \mu \mathrm{m}$ on the entrance plane of the slit when the slit is placed $80 \mathrm{~cm}$ from the cylindrical glass. The slit width was set at $50 \mu \mathrm{m}$. A phase change in the He-Ne probe beam due to the arrival of the shock front will alter the deviation shift of the fringes.

\section{Experimental Results}

As we have reported in our previous works [15-27], the secondary plasma and the primary plasma could be clearly observed by the naked eye in surrounding air below 10 Torr. The secondary plasma exhibited a characteristics hemispherical shape with brilliant colors associated with emissions from the constituent atoms, while the primary plasma displayed an intense white color associated with the continuum emission. The hemispherical shape of the secondary plasma was indicative of the dominant role of the shock wave in its generation, connected with the typical hemispherical shape of the shock wave.

In the present experiment which is conducted in surrounding air pressure of 760 Torr, the secondary plasma appears to be dominated by strong white continuum from the primary plasma and exhibits a cone-like structure instead of the hemispherical shape common to shock wave induced plasma as shown in Fig. 2. We note further that the secondary plasma can still be readily distinguished 
from the primary plasma by the naked eye even in such high pressure by the presence of indigo color (a mixture between magenta color of zinc emission and white continuum emission). However, the highly dense white color region of the primary plasma expands accordingly and blurring the boundary between the two plasma regions. Nevertheless, the presence of secondary plasma can still be clearly detected by time-resolved spectroscopic measurements, even with surrounding air at 760 Torr. Whereas only a high-intensity continuum emission spectrum is detected in a region restricted around the primary plasma, distinct atomic lines become clearly observable as the detection region is shifted $2 \mathrm{~mm}$ off the primary plasma and after a considerable delay (of the order of microseconds) with respect to the irradiation pulse. It is amply confirmed therefore that the laser induced plasma still consists of a primary as well as secondary parts even at a pressure as high as 760 Torr.

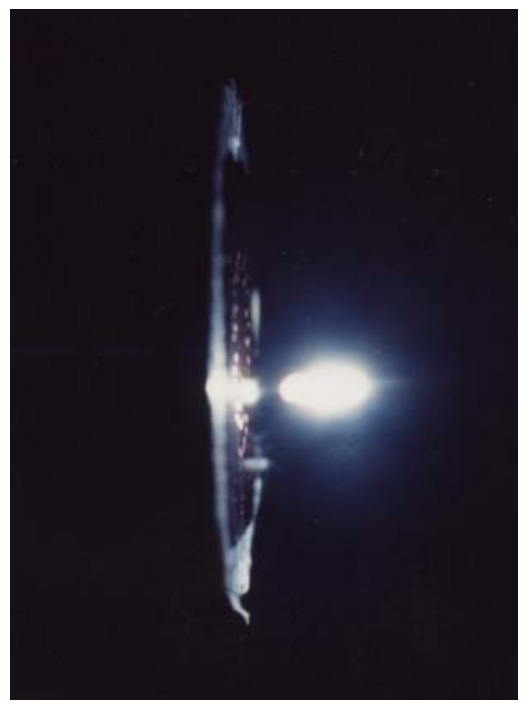

Figure 2 Photograph of the plasma generated by tight focusing $26 \mathrm{~mJ} \mathrm{Nd}-\mathrm{YAG}$ laser onto zinc sample surface under air pressure of 760 Torr.

Another feature supporting the occurrence of a shock wave is the density jump associated with the presence of a thin shell structure at the shock wave front. The observation of this density jump has been reported by Basov et al [28-29] from their experiments using a shadowgraph technique and a Schilieren photograph technique, consecutively. The detection of a density jump was also reported at about the same time and independently by Bobin et al [30], Hall [31], and Emmony et al [32], employing an image converter camera in their recording of the expanding plasma and the detection of emission fronts. These 
evidences were further confirmed by the observation made by Hohla et al [33], using a shadowgraph technique.

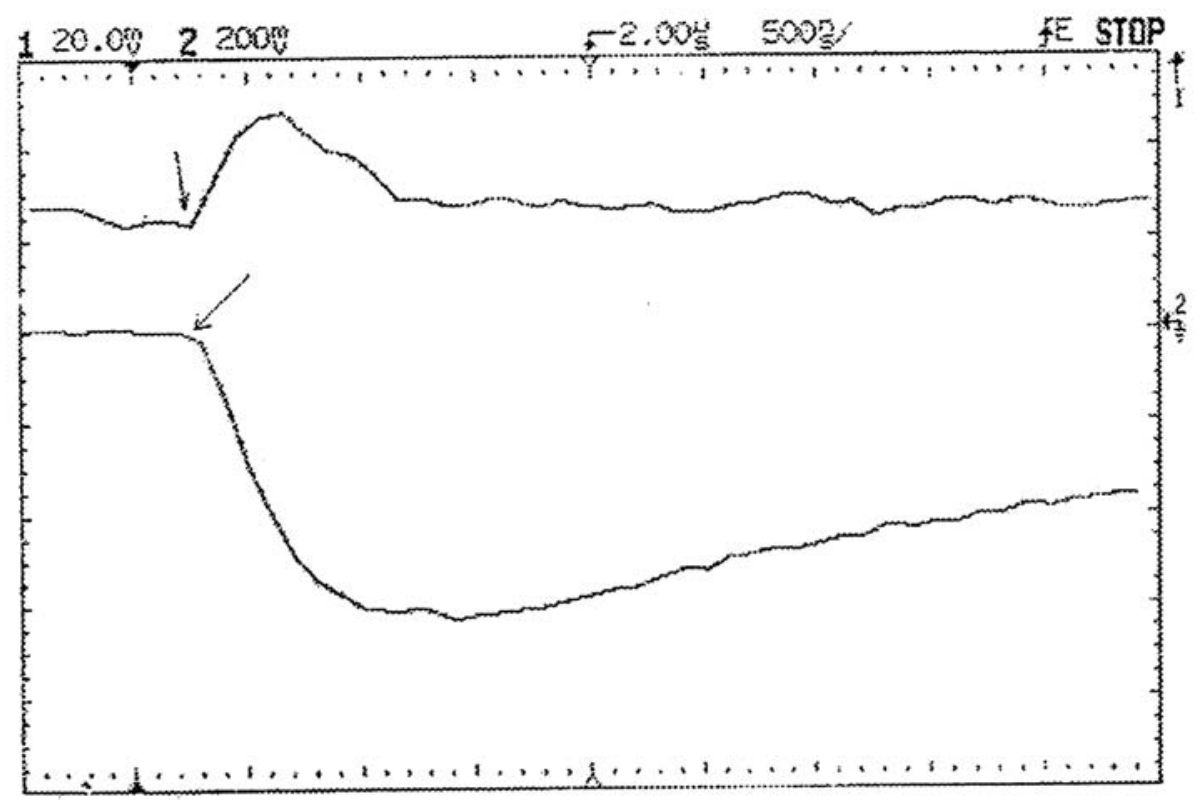

Figure 3 The appearance of the density jump (upper curve) and the emission intensity of the $\mathrm{Zn} \mathrm{I} 481.0 \mathrm{~nm}$ (lower curve) line at $2 \mathrm{~mm}$ above the sample surface. The air pressure was kept constant at 760 Torr. The laser irradiation was defocused at $-3 \mathrm{~mm}$ onto zinc sample surface.

Fig. 3 shows the result of the simultaneous detection of the density jump and the plasma emission of zinc neutral line ( $\mathrm{Zn} \mathrm{I} 481.0 \mathrm{~nm}$ ) at $2 \mathrm{~mm}$ probing position. The plasma is generated by a Nd-YAG laser of $26 \mathrm{~mJ}$, which is defocused at -3 $\mathrm{mm}$ onto the zinc sample in air at 760 Torr. The power density under this condition was estimated to be around $2 \mathrm{GW} / \mathrm{cm}^{2}$. From the above figure it is seen that the occurrence of the emission intensity of $\mathrm{Zn} \mathrm{I} 481.0 \mathrm{~nm}$ took place at the same times as the arrival of the shock waves as pointed out by the arrow in Fig. 3. This coincidence was also confirmed when the time sensitivity of the scope was set at $200 \mathrm{~ns}$. It is further revealed that the density jump at $2 \mathrm{~mm}$ above the zinc sample surface took place around $200 \mathrm{~ns}$ after the laser pulse.

Fig. 4 shows the propagation characteristics of the shock front and emission front of Zn I $481.0 \mathrm{~nm}$. The plasma is generated by a Nd-YAG laser of $26 \mathrm{~mJ}$, which is defocused at $-3 \mathrm{~mm}$ onto the zinc sample in air at 760 Torr, while Fig. 5 shows the propagation characteristics of the shock front and emission front of $\mathrm{Zn} \mathrm{I} 481.0 \mathrm{~nm}$ when the plasma is generated by a Nd-YAG laser of $26 \mathrm{~mJ}$, 
which is tight focused onto the zinc sample in air at 760 Torr. These data were collected in the same manner as the case of Fig. 3. All the curves exhibit two linear segments with different slopes. As we have mentioned above, it is remarkably that the coincidence between the emission front and shock front is clearly sustained, as far as $4 \mathrm{~mm}$ from the sample surface (Fig. 4) at $-3 \mathrm{~mm}$ defocused condition, and up to $5 \mathrm{~mm}$ from the sample surface at tight focused condition (Fig. 5), before the curves corresponding to the two fronts branch off. These observations constitute a rather convincing evidence for our shock wave plasma model as described earlier. [25-26]

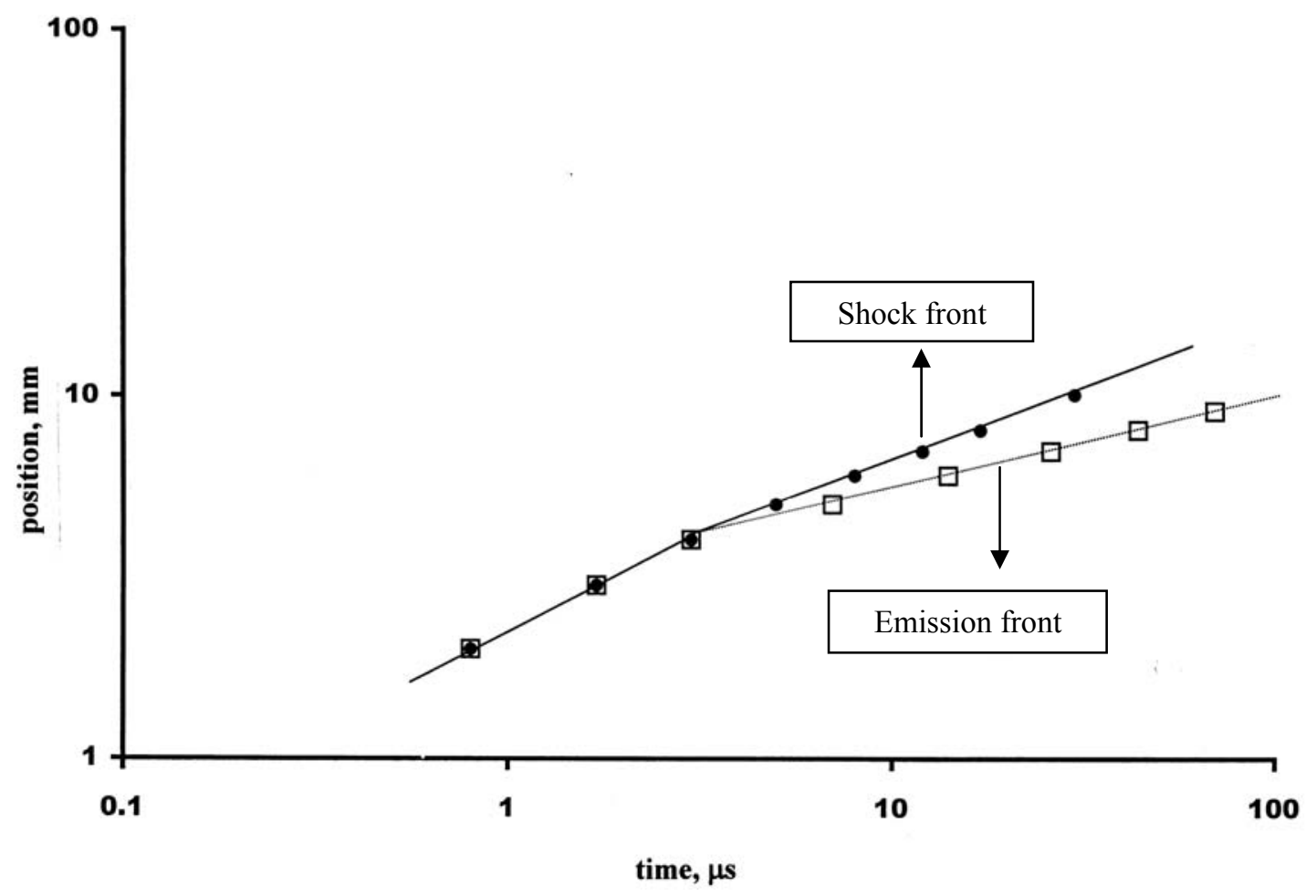

Figure 4 Correlation between the arrival of the shock front and emission front as a function of time at defocused $-3 \mathrm{~mm}$ condition.

\section{Discussion}

In a series of subsequent time-resolved experiments using a carbon-dioxide laser [21-22], an excimer laser [18] and a Nd-YAG laser [17,25], we demonstrated that this secondary plasma was excited by the shock wave while the primary plasma acted as an initial explosion energy source. We have offered 
a theory with respect to the excitation mechanism of the secondary plasma as follows. Right after the cessation of the primary plasma, atoms gushed out from the primary plasma at supersonic speed. It is assumed that the surrounding gas plays the role of a damping material, impeding the free expansion of the propelling atoms, by forming something like a wall against which compression is taking place. As a result of this compression, a shock wave is generated in the surrounding gas. The most important point of the shock wave model is that the energy required to produce the secondary plasma is supplied in the form of kinetic energy from the propelling atoms. By means of this compression, the kinetic energy of the propelling atoms is converted into thermal energy in the plasma, by which atoms are excited.

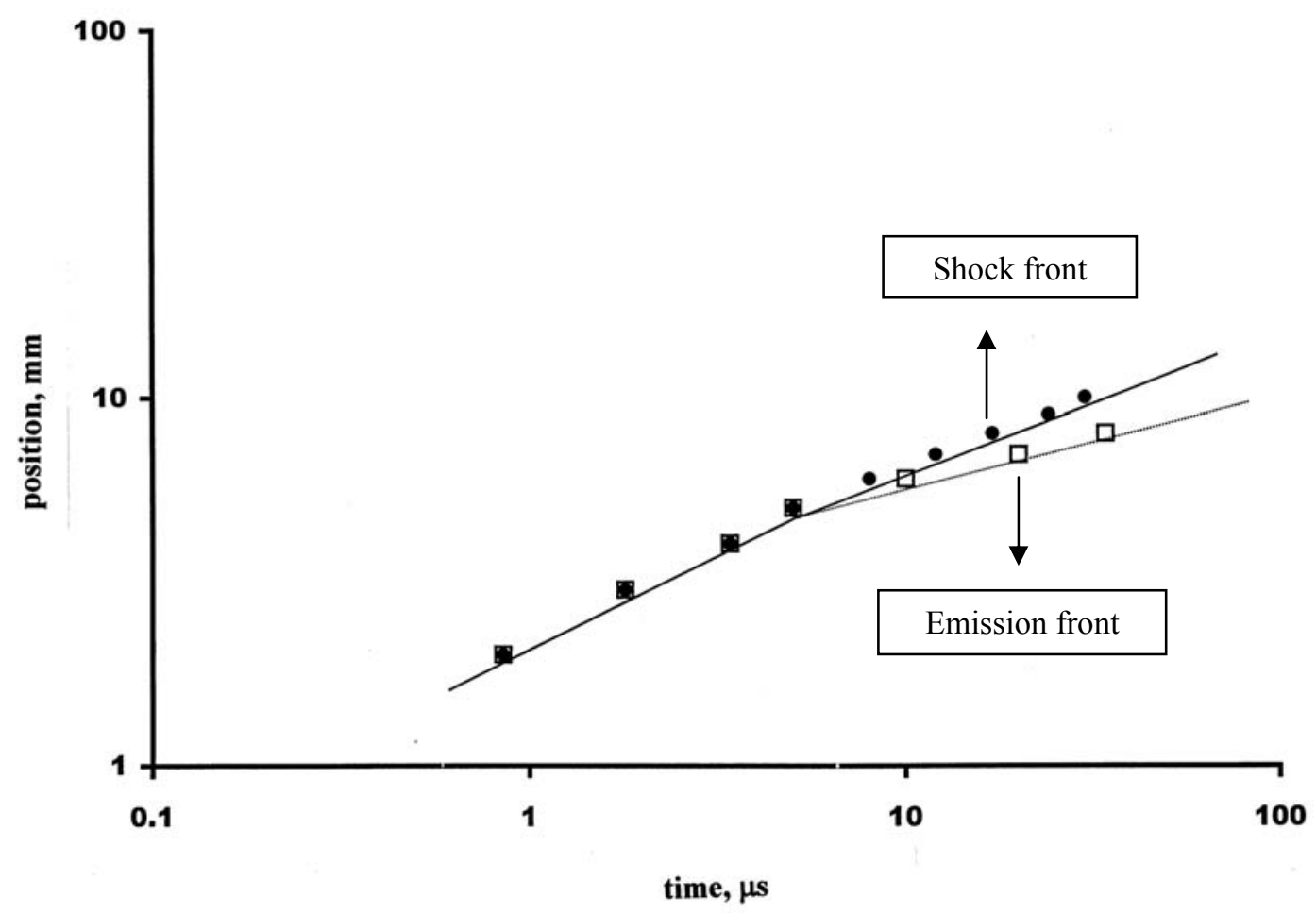

Figure 5 Correlation between the arrival of the shock front and emission front as a function of time at tight focused condition.

In a different experiment carried out recently [26], we proposed that, at the initial stage of the secondary plasma expansion, the front of the propelling atoms and the front of the shock wave coincided and moved together with time. However, at a later stage, the front of the atom cluster was separated from that of the shock wave induced in the surrounding gas. In order to further explain the characteristics of the secondary plasma, we presented a hypothetical model, in 
which the dynamical process of the secondary plasma was separated into two stages - namely, the shock excitation stage, which was followed by the cooling stage. Thus in the shock excitation region, atoms were excited by the heat arising from strong compression between the shock front and propelling atoms. The cluster of propelling atoms was supposed to move in a manner somewhat like a piston pushing the surrounding gas. We called this stage the shock excitation stage. Soon afterwards, the cluster of atoms began to slow down while losing its energy to the surrounding gas. As a result, no further excitation took place, since the compression between the shock front and propelling atoms could no longer be sustained due to the widening separation between them, while the cluster of atoms continued to move forward with its residual momentum. Since the surrounding gas behind the shock wave was left with high temperature, and the secondary plasma also contained large amount of residual thermal energy, the cooling of the plasma was bound to proceed slowly with time. We have called this part of the process the cooling stage.

Complete illustrations of the above phenomena are given in Figs. 4 and 5 using zinc sample. It is seen that, at 760 Torr air pressure and $-3 \mathrm{~mm}$ defocused condition, the separation begins to take place at $3 \mu \mathrm{s}$, while for the case of tight focused condition, the separation begins to take place at $5 \mu \mathrm{s}$. This observation means that the excitation stage last about $3 \mu$ s at defocused condition, and about $5 \mu \mathrm{s}$ at tight focused condition. The time span of $3 \mu \mathrm{s}$ and $5 \mu \mathrm{s}$ for $-3 \mathrm{~mm}$ defocused and tight focused condition, as mentioned earlier, indicating that the shock excitation process lasts longer for the case of higher laser power density as we have reported in our previous work.[24]

Sedov [34] has derived a theoretical relationship between the propagation time $(t)$ of the shock wave and the distance of its front, $r$, from the position of the source of explosion as follows:

$$
r=(E o / \alpha \rho)^{1 / 5} t^{2 / 5}
$$

where Eo is the initial explosion energy, $\rho$ is the density of the surrounding gas, and $\alpha$ is the constant involving $\gamma$ (the ration of specific heats of the gas). Also it is well known that for a detonation wave [35-36], $r$ is proportional to $t^{3 / 5}$. The slopes of the curves shown in Figs. 4 and 5, 0.6 in the excitation stage region and 0.4 in the cooling stage region for the emission front are in good agreement with the values expected from the above Sedov equation.

\section{Conclusion}

We have shown in this experiment that laser plasma generated by Nd-YAG laser irradiation on zinc sample in atmospheric air pressure, generally exhibits 
features of secondary plasma well known in low pressure plasma. Analysis of the dynamical characteristics has led us to the conclusion that models other than the shock wave model, such as the gas breakdown model, should be excluded, at least for a zinc sample, as not satisfactorily explaining the excitation process in the secondary plasma generated at atmospheric air pressure of 760 Torr.

\section{References}

1. $\quad$ Brech, F. \& Cross, L., Appl. Spectrosc. 16 (1962) 59.

2. $\quad$ Cremers, D. A., Appl. Spectrosc. 41 (1987) 572.

3. Yamamoto, K. Y., Cremers, D. A., Ferris, M. J. \& Foster, L. E., Appl. Spectrosc. 50 (1996) 222.

4. Lorenzen, C. J., Carlhoff, C., Hahn, U. \& Jogwich, M., J. Anal. Atom. Spectrom. 7 (1992) 1029.

5. Majidi, V. \& Joseph, M. R., Crit. Rev. Anal. Chem. 23 (1992) 143.

6. Multari, R. A., Foster, L. E., Cremers, D. A. \& Ferris, M. J., Appl. Spectrosc. 50, 12 (1996) 1483.

7. Radziemski, L. J., Microchem. J. 50, 3 (1994) 218.

8. $\quad$ Song, K., Lee, Y. I. \& Sneddon, J., Appl. Spectrosc. 51, 3 (1997) 183.

9. $\quad$ Ahmad B. J. \& Goddard, J., Fiz. Mal. 14, 43 (1993) 43.

10. Piepmeier, E. H. \& Osten, D. E., Appl. Spectrosc. 25 (1971) 642.

11. Dimitrov, G. \& Gagov, V., Spectrosc. Lett. 10 (1979) 337.

12. Gibson, A. F., Hughes, T. P. \& Ireland, C. L. M., J. Phys. D: Appl. Phys. 4 (1971) 1527.

13. Kuzuya, M. \& Mikami, O., Jpn. J. Appl. Phys. 29 (1990) 1568.

14. Iida, Y., Spectrochim. Acta B45 (1990) 1353.

15. Kagawa, K., Yokoi, S. \& Nakajima, S., Spectrochim. Acta B37 (1982) 789.

16. Kagawa, K., Yokoi, S. \& Nakajima, S., Opt. Commun. 45 (1983) 261.

17. Setia Budi, W., Baskoro, E. T., Pardede, M., Kurniawan, H., Tjia, M.O. \& Kagawa, K., Appl. Spectrosc. 53, 11 (1999) 1347.

18. Kagawa, K., Kawai, K., Tani, M. \& Kobayashi, T., Appl. Spectrosc. 48 (1994) 198.

19. Kurniawan, H., Nakajima, S., Batubara, J. E., Marpaung, A. M., Okamoto, M. \& Kagawa, K., Appl. Spectrosc. 49, 8 (1995) 1067.

20. Tani, M., Kurniawan, H., Ueda, H., Mizukami, K., Kawai, K. \& Kagawa, K., Jpn. J. Appl. Phys. 32 (1993) 3838.

21. Kurniawan, H., Tjia, M. O., Barmawi, M., Yokoi, S., Kimura, Y. \& Kagawa, K., J. Phys. D: Appl. Phys. 28 (1995) 879.

22. Kurniawan, H., Kobayashi, T. \& Kagawa, K., Appl. Spectrosc. 46, 4 (1992) 581.

23. Kurniawan, H., Ishikawa, Y., Nakajima, S. \& Kagawa, K., Appl. Spectrosc. 51, 12 (1997) 1769. 
24. Setia Budi, W., Baskoro, E. T., Pardede, M., Kurniawan, H., Tjia, M.O. \& Kagawa, K., Appl. Spectrosc. 53, 11 (1999) 1347.

25. Setia Budi, W., Suyanto, H., Kurniawan, H., Tjia, M.O. \& Kagawa, K., Appl. Spectrosc. 53, 6 (1999) 719.

26. Kurniawan, H., Lahna, K., Lie, T. J., Kagawa, K. \& Tjia, M. O., Appl. Spectrosc. 55, 1 (2001) 92.

27. Kurniawan, H., Lie, T. J., Idris, N., Tjia, M. O., Ueda, M. \& Kagawa, K., J. Spectrosc. Soc. Jpn. 50, 1 (2001) 13.

28. Basov, N. G., Krokhin, O. N. \& Skizkov, G. V., JETP Lett., 6 (1967) 168.

29. Basov, N. G., Gribkov, V. A., Krokhin, O. N. \& Skizkov, G. V., Sov. Phys. - JETP 27 (1968) 575.

30. Bobin, J. L., Durand, Y. A., Langer, P. \& Tonon, G., J. Appl. Phys. 39 (1968) 4184.

31. Hall, R. B., J. Appl. Phys. 40 (1969) 1941.

32. Emmony, D. C. \& Irving, J., Br. J. Appl. Phys. 2 (1969) 1168.

33. Hohla, K., Buchi, K., Wienecke, R. \& Wrkowiski, S., Z. Naturf. A 24 (1969) 1244.

34. Sedov, L. i., Similarity and Dimensional Methods in Mechanics (Academic Press, New York and London, 1959) p.213.

35. Ramsden, S. A. \& Savic, P., Nature, 19 (1964) 1217.

36. Daiber, J. W. \& Thompson, H. M., the Physics of Fluids, 6 (1967) 1162. 